\title{
Understanding the Glycoproteins Release from Alginate-Barium Capsules in Physiologic Enviroments
}

\author{
Edgar Perez Herrero, Eva M. Martin del Valle*, Miguel A. Galán \\ Department of Chemical Engineering, University of Salamanca, Salamanca, Spain \\ E-mail:"emvalle@usal.es \\ Received July 5, 2011; revised July 27, 2011; accepted August 12, 2011
}

\begin{abstract}
The authors carried out a steady and unsteady mass transfer studies to simulate both the release of proteins in physiologic environments and proteins transport through a tissue or organ from polymeric capsules by using a substance, the rhodamine B isothiocyanate dextran (RBID) that mimics the behaviour of glycoproteins such as vascular endothelial growth factor (VEFG). These studies highlighted the importance of electrostatic interactions between alginate and proteins in the release processes. Thereby, this fact has opened new perspectives in order to use these kinds of capsules in protein recognition processes. The electrostatic interactions between alginate and RBID allow $\mathrm{pH}$-dependent controlled release systems that simulate the behaviour of glycoproteins.
\end{abstract}

Keywords: Controlled Release Systems, Proteins, Glycoproteins, Growth Factors, Mass Transfer, Unsteady, Steady

\section{Introduction}

The most common methods of drug administration, tablets or pills and injections, satisfy the requirements on efficacy for traditional drugs, not being suitable for drugs developed in recent years, such as drugs based on proteins. These difficulties lead to the need of developing new systems of drug delivery, such as encapsulation, which maintain therapeutic levels of the drug without producing the unwanted side effects. In general, the main advantages of these new systems are: providing protection to the encapsulated material and to enable constant release profiles over time, without repeated administration [1].

The key point in the administration of traditional drugs is to maintain the blood level of the active agent between a maximum value, which represents the toxic level, and a minimum level below which the drug is not effective. In the controlled drug delivery systems, like microcapsules, the drug level in blood follows a constant profile between the minimum and maximum levels allowed, over a long period of time [2].

In order to achieve the understanding of the real behaviour of the capsules in the human body for a later use in any of the multiple applications in the field of the medicine is needed to carry out studies of mass transfer.
To do that it has been simulated the release of proteins from polymeric spheres into physiologic environments (external medium of finite volume) such as eyes [3-5], bones [6,7], and periodontal pockets [8-10] by means of a steady mass transfer study. This sort of protein release process may occur when the protein concentration in the medium surrounding the drug delivery device builds up to appreciable levels. But also, it has been developed an unsteady mass transfer study to simulate the protein transport through a tissue or organ after a therapeutic leakage from storage cavities.

Macroscopically, both the physiological environments and tissues or organs, are ideally assumed as an isotropic porous medium as shown in Figure 1 [11], since it is studied the release of proteins from capsules to a packed bed, simulated by the other capsules.

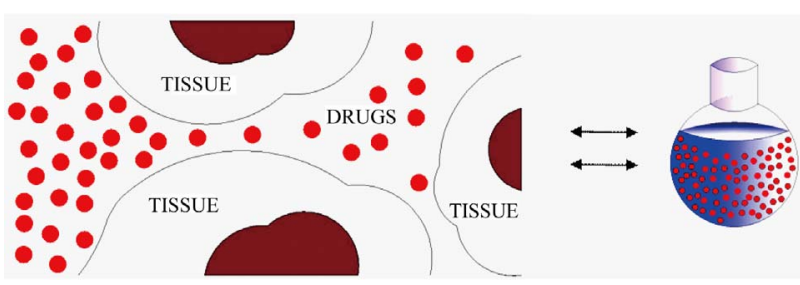

Figure 1. Drug transport in the human body, assumed as an isotropic porous medium. 
To do that, in this paper, a novel and simple method was used in order to develop the steady and unsteady mass transfer studies. A simple perturbation method based on the use of a colored substance, rhodamine B isothiocyanate dextran (RBID), was used to mimic the release of glycoproteins from polymeric capsules.

RBID is an amphoteric dye that is normally listed as basic due to its overall positive charge. The structure of the rhodamine B is shown in Figure 2. The main feature of the structure of this dye is the quaternary nitrogen, but it is also necessary to take into account the existence of a carboxyl group, which would give the molecule an amphoteric character at high $\mathrm{pH}$ values [12].

In addition, RBID is a biomacromolecule available in three molecular weights, 10,40 , and $70 \mathrm{KDa}$, due to the dextran molecules. Dextran is a complex, hydrophilic, branched polysaccharide made of many chains of glucose, characterized by its high molecular weight (MW), good water solubility, low toxicity, and relative inactivity [13].

Rhodamine B molecules can be functionalized with an isothiocyanate group $(-\mathrm{N}=\mathrm{C}=\mathrm{S})$, replacing a hydrogen atom on the upper ring of the structure (see Figure 3). The dextran molecule binds to the rhodamine B molecule by the isothiocyanate group, leaving free the carboxyl group (Figure 3). And then, RBID maintain the same effective charge that rhodamine B, because of RBID retains the carboxyl group that gives the amphoteric character to the molecule at high $\mathrm{pH}$ values (Figure 3).

\section{Materials and Methods}

\subsection{Materials}

Sodium alginate from macrocystis pyrifera (medium vis-<smiles>CCN(CC)c1ccc2c(c1)Oc1cc([N+](CC)(CC)CC)cc(c1-c1ccccc1C(=O)O)O2</smiles>

Figure 2. Rhodamine B structure.

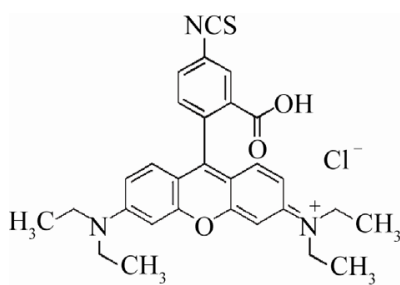

(a)

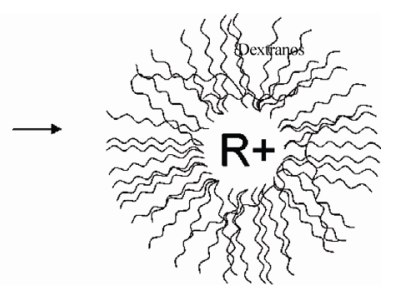

(b)
Figure 3. Structure of the Rhodamine B isothiocyanate (a) and RBID (b). cosity) was purchased from Sigma Chemicals, barium chloride dihydrate, reagent grade was purchased from Scharlau, RBID was purchased from Sigma Chemicals.

\subsection{Production of Capsules}

RBID-alginate solutions with different concentrations of alginate $(1.2 \mathrm{wt} \%$ and $3 \mathrm{wt} \%)$ were prepared dissolving the proper amount of alginate in $1 \mathrm{mg} / \mathrm{mL}$ of RBID solution. Alginate-RBID solutions were extruded drop by drop through a $25 \mathrm{G}$ needle using a sterile syringe into a 2 $\mathrm{wt} \%$ barium chloride solution under stirring speed of 30 rpm. After two minutes of gelation, capsules were filtrated.

\subsection{Experimental Device to Steady Mass Transfer Study}

In order to simulate the steady diffusional phenomenon, one gram of the capsules generated were placed in a 25 $\mathrm{mL}$ spherical batch reactor with $5 \mathrm{~mL}$ of deionised water. The reactor was placed over an orbital sake rotator with a constant speed of 110 RPM. This stirring speed attempts to simulate the movement of the human body in the process of releasing the protein in a physiological medium.

\subsection{Experimental Device to Unsteady Mass Transfer Study}

In order to simulate the phenomenon at the organ or tissue, one gram of capsules were placed in a $25 \mathrm{~mL}$ continuous flow reactor with $5 \mathrm{~mL}$ of deionised water. The reactor was placed over an orbital rotator with in constant speed of $110 \mathrm{rpm}$. This stirring speed attempts to simulate the movement of the human body in the process of releasing the protein through a tissue or organ.

Two peristaltic pumps keep constant the volume inside the reactor in addition to providing the advection effect to the process. The amount of RBID released was determined by means of a spectrophotometer at $555 \mathrm{~nm}$ (maximum peak absorbance for RBID) connect to the system providing continuous data every minute. The scheme of the equipment used to the simulation is shown in Figure 4.

\section{Experimental Mass Transfer Study}

The cumulative release data of three different molecular weight of RBID (10,000, 40,000, and 70,000 Da) were obtained and plotted in Figures 5 and $\mathbf{6}$ to the steady and unsteady processes, respectively. These data were obtained for two different alginate concentrations $(1.2 \mathrm{wt} \%$ and $3.0 \mathrm{wt} \%$ ). 


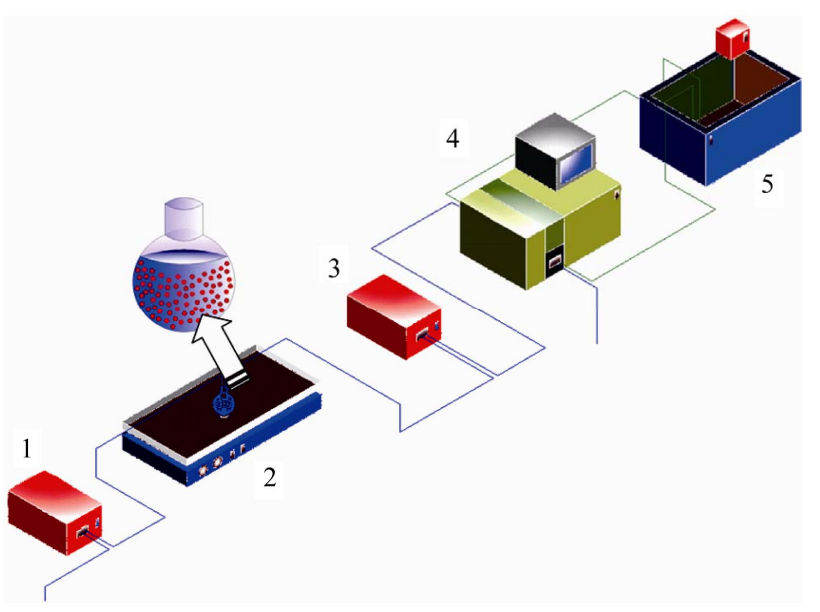

Figure 4. Equipment used to simulate the unsteady mass transfer phenomena. 1 and 3 peristaltic pumps; 2 reactor over an orbital shake rotator; 4 spectrophotometer; 5 thermostatic bath.

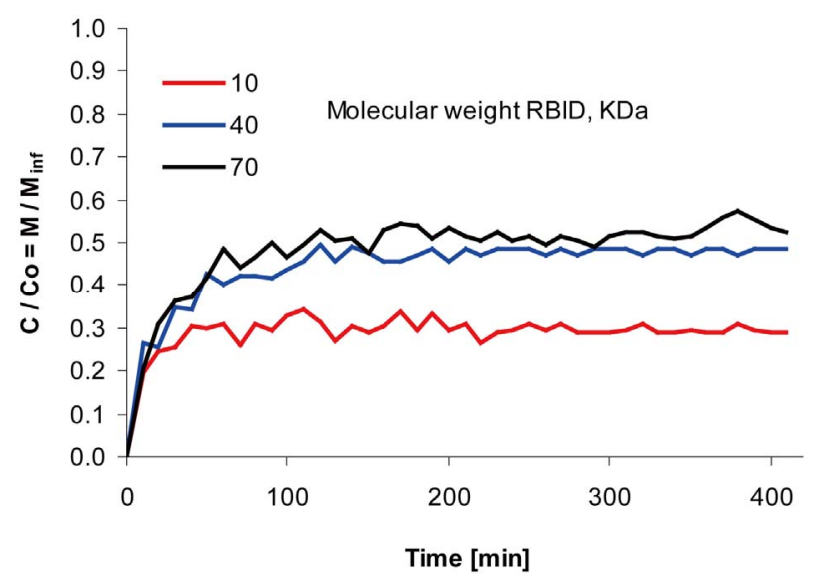

(a)

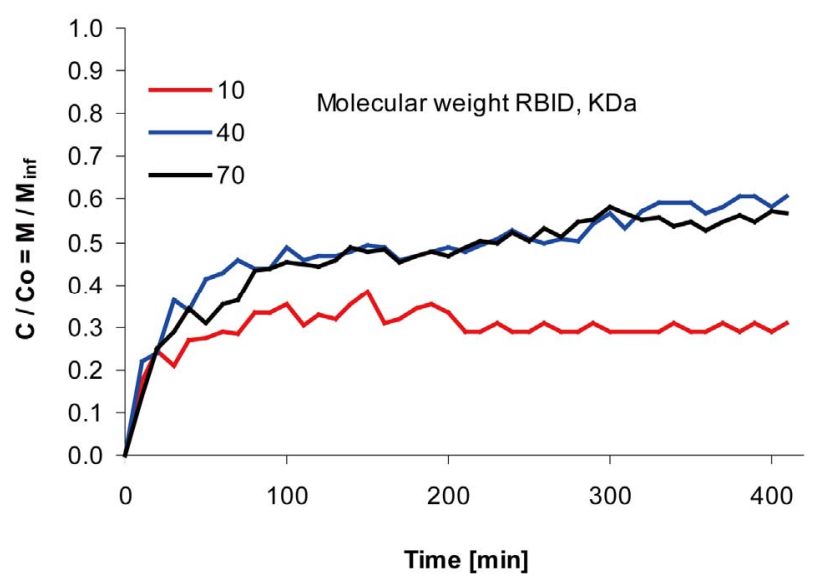

(b)

Figure 5. Cumulative steady release of RBID at different molecular weight with different alginate concentration. (a) $1.2 \mathrm{wt} \%$; (b) $3.0 \mathrm{wt} \%$.
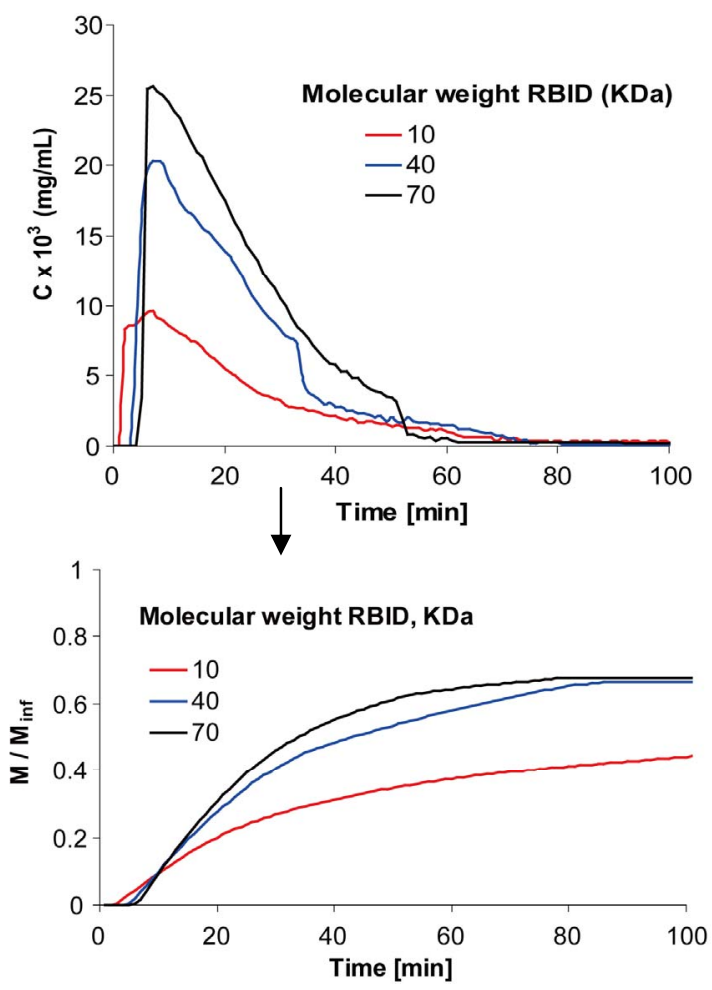

(a)
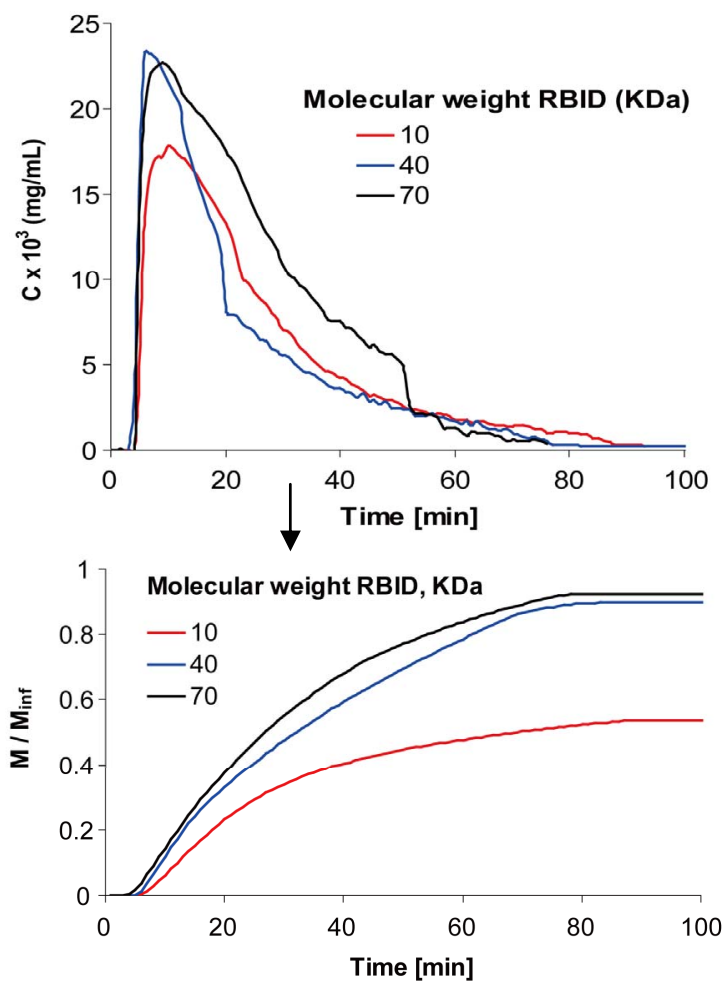

(b)

Figure 6. (a) Cumulative unsteady release of RBID at different molecular weight (Alginate concentrat.: $1.2 \mathrm{wt} \%$ ); (b) Cumulative unsteady release of RBID at different molecular weight (Alginate concent.: $3.0 \mathrm{wt} \%$ ). 
In these figures, the cumulative release of RBID has been adimensionalized by dividing by the amount initially loaded in the capsules. Therefore, it is possible to compare the data from the three RBID molecular weights.

From Figures 5 and $\mathbf{6}$ it can be seen that the smallest RBID exhibits a much lower cumulative release in both alginate concentrations despite its smaller molecular weight. Also, it is showed that high alginate concentration $(3.0 \mathrm{wt} \%)$ leads to higher cumulative release. Both behaviors can be observed quantitatively in Tables $\mathbf{1}$ and $\mathbf{2}$.

\subsection{Effect of Molecular Weight of RBID in the Cumulative Release of RBID}

From the data shown it can be seen a concurrent release of the molecules encapsulated since the smallest RBID exhibits a much lower cumulative release in both alginate concentrations despite its smaller molecular weight.

This behavior can be assigned to the presence of attractive electrostatic interactions (Figure 7) between the protonated amine group of RBID (positive charged) and carboxyl groups of alginate (negative charged), that are directly proportional to the charges, and indirectly proportional to the square of the distance between charges, i.e.,

$$
f=K\left(q q^{\prime}\right) / d^{2}
$$

where $K$ is the Coulomb constant.

As it was mentioned before, the variation of the molecular weight of the RBID is due to the dextran molecules size. In this work, it was used three different molecular weight of RBID (10000, 40000, and $70000 \mathrm{Da})$. RBID is a core, the rhodamine $\mathrm{B}$, bond to the dextran chains by means of the isothiocyanate group. The larger size of dextran molecules, the smaller the attractive electrostatic interactions due to sterically hinder of the dextran molecules because of its conformation that separates with a greater distance, $d$, the positive and negative charges (Figure 8). The dextran molecules have a ran-

Table 1. Cumulative release data (steady process).

\begin{tabular}{|c|c|c|c|}
\hline \multirow{3}{*}{$\begin{array}{c}\text { Alginate } \\
\text { concentration } \\
(w \mathbf{w} \%)\end{array}$} & \multicolumn{3}{|c|}{ Molecular weight of RBID (KDa) } \\
\hline & 10 & 40 & 70 \\
\hline & \multicolumn{3}{|c|}{ Cumulative release, $\mathrm{C} / \mathrm{Co}=\mathrm{M} / \mathrm{M}_{\infty}(\%)$} \\
\hline 1.2 & 28 & 49 & 52 \\
\hline 3.0 & 31 & 61 & 57 \\
\hline
\end{tabular}

Table 2. Cumulative release data (unsteady process).

\begin{tabular}{|c|c|c|c|}
\hline \multirow{3}{*}{$\begin{array}{c}\text { Alginate concentration } \\
(\mathbf{w t} \%)\end{array}$} & \multicolumn{3}{|c|}{ Molecular weight of RBID (KDa) } \\
\hline & 10 & 40 & 70 \\
\hline & \multicolumn{3}{|c|}{ Cumulative release, $\mathbf{M} / \mathbf{M}_{\infty}(\%)$} \\
\hline 1.2 & 44 & 67 & 68 \\
\hline 3.0 & 54 & 90 & 92 \\
\hline
\end{tabular}

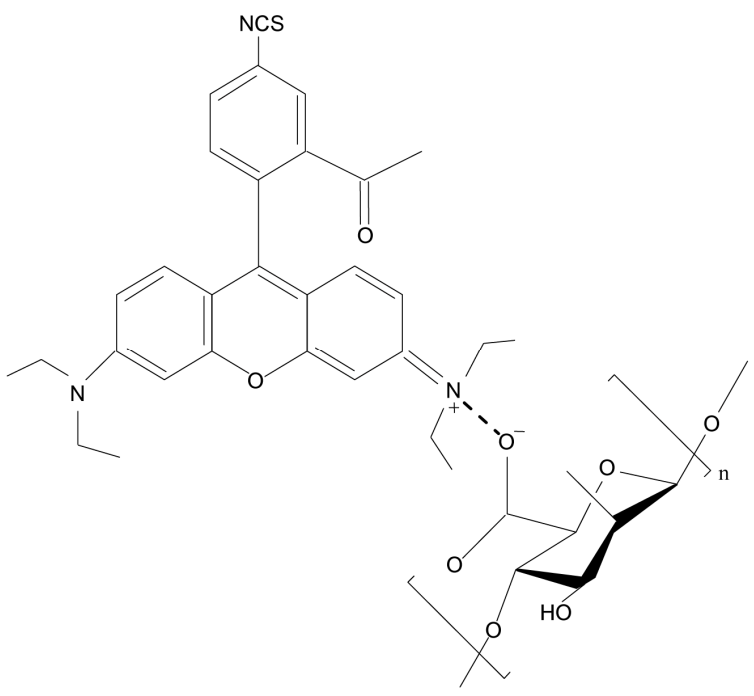

Figure 7. Electrostatic interactions between RBID and alginate.

dom-coiled conformation that covers the RBID core to a greater or lesser extend, depending on the size of the dextran molecules, hindering the electrostatic interactions (Figure 8).

Therefore, the larger RBID molecules will be less attracted to the alginate because of, based on the Equation (1), the distance between charges will be longer, and therefore there will be a larger cumulative release of high molecular weight RBID $[14,15]$.

This fact proves that the number of free molecules of RBID in the capsules increases as the electrostatic interactions decrease. So, it is as if the effective concentration for the same initial concentration of RBID was greater with increasing molecular weight of RBID. The continuous experiments should be taken into account for the quantification of the electrostatic interactions between RBID and alginate. Figures 6(a) and 6(b) showed that the concentration of the supernatant is zero after finishing the overall process. There are not free molecules capable of being released from the capsules at the end of the process, and therefore the RBID molecules that do not release from capsules will be linked by electrostatic bonds to the alginate molecules. So, from a mass balance to the capsules it is possible to quantify the electrostatic bonds that are listed in the Table 3, where it is also shown a summary of the influence of electrostatic interactions in the cumulative release of RBID.

Table 3 shows that by increasing the molecular weight of RBID, for the two concentrations of alginate, the electrostatic interactions between alginate and RBID decrease, that is, when the molecular weight of RBID is increased, the free molecules of RBID is also increased into the capsules, increasing the cumulative release of RBID. 


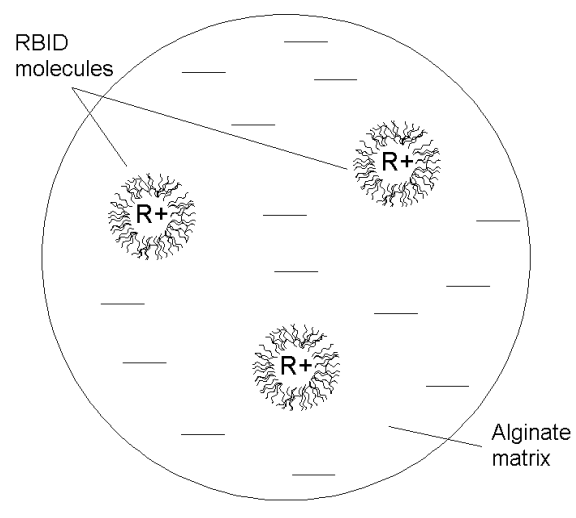

(a)

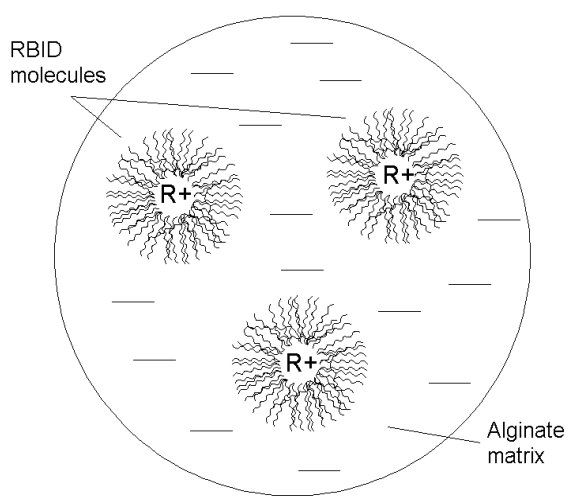

(b)

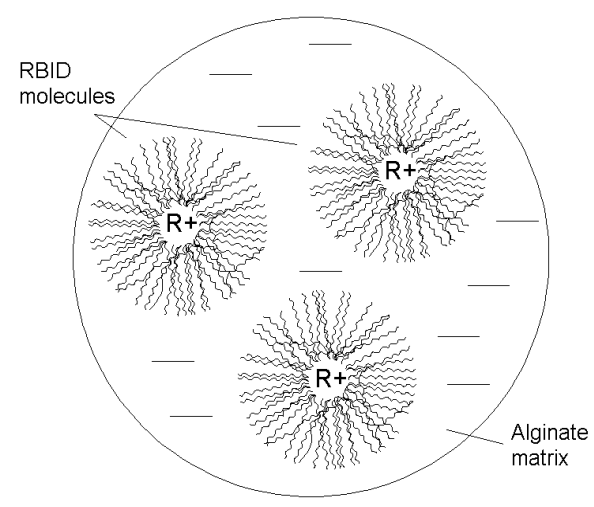

(c)

Figure 8. Simulation of the conformation of RBID molecules in the capsules. Molecular weight of RBID: (a) 10 KDa, (b) 40 KDa, (c) 70 KDa.

The behavior found for RBID molecules opens new ways of application for the capsules, taking into account that the $\mathrm{pK}_{\mathrm{a}} \mathrm{s}$ of the RBID molecules, unknown at the present time, will in turn determine the charge and the acid-base behavior of the isomers as a function of $\mathrm{pH}$. At higher $\mathrm{pH}$ values, the attraction of RBID to the alginate may be diminished as a result of unfavorable electrostatics interactions when the carboxyl group gives the molecule the amphoteric character. Knowing this, it is possible to achieve a $\mathrm{pH}$-dependent controlled release of
Table 3. Effect of the electrostatic interactions in the cumulative release of $\mathrm{RBID}$.

\begin{tabular}{|c|c|c|c|c|c|}
\hline & \multicolumn{3}{|c|}{$\begin{array}{c}\text { Molecular weight of } \\
\text { RBID (KDa) }\end{array}$} \\
\hline & & & $\mathbf{1 0}$ & 40 & 70 \\
\hline \multirow{2}{*}{\multicolumn{3}{|c|}{$\begin{array}{l}\text { Electrostatic interactions } \\
\text { Cumulative release }\end{array}$}} & $\gg>$ & $>$ & $>$ \\
\hline & & Cumulative release & + & ++ & +++ \\
\hline \multirow{2}{*}{$\begin{array}{l}\begin{array}{l}\text { Alginate } \\
\text { concentration } \\
(\mathbf{w t} \%)\end{array} \\
\end{array}$} & 1.2 & \multirow{2}{*}{$\begin{array}{l}\text { \% Electrostatic } \\
\text { interactions }\end{array}$} & 56 & 33 & 32 \\
\hline & 3.0 & & 46 & 10 & 8 \\
\hline
\end{tabular}

proteins, resulting in protein imprinting processes.

This behaviour of RBID makes this macromolecule very similar to proteins. But, in particular, RBID mimics the behaviour of a special type of protein, the glycoproteins. These substances are proteins (core) that contain oligosaccharide chains (glycans) covalently attached to their polypeptide side-chains. Then, the structure of the glycoproteins is very similar to the RBID, that is, RBID is a core, the rhodamine $\mathrm{B}$, bond to the dextran chains by means of the isothiocyanate group (see Figure 9).

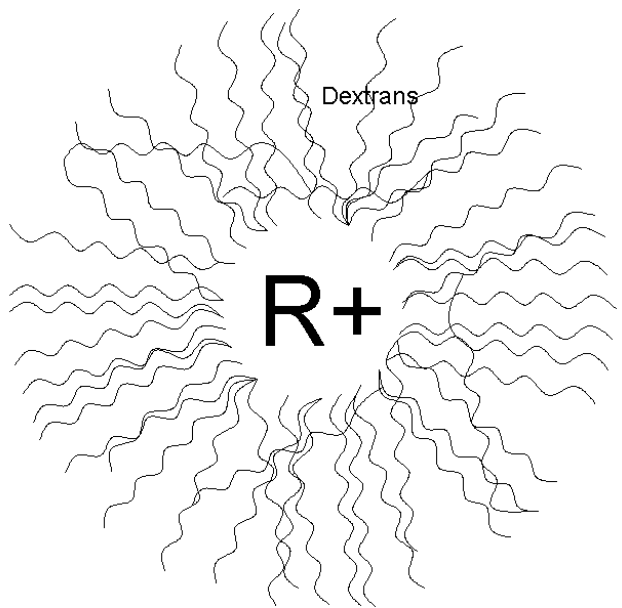

(a)

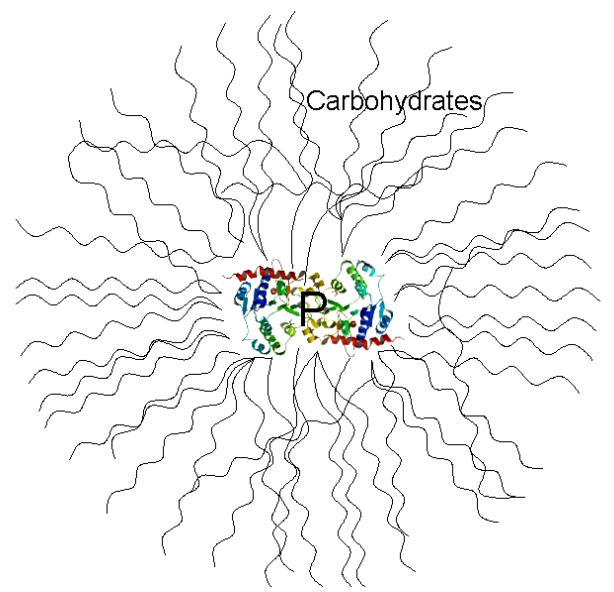

(b)

Figure 9. Analogy between RBID and Glycoprotein molecules. (a) RBID; (b) Glycoprotein. 
Several hormones, antibodies, various enzymes, receptor proteins, cell adhesion proteins, growth factors, etc, are glycoproteins. This fact is very important because it is possible to study the controlled release of several growth factors by means of RBID that is much cheaper than growth factors but also mimics the behaviour of these proteins. Herrero et al. [16] achieved the immobilization of mesenchymal stem cells and monocytes in biocompatible microcapsules to cell therapy. Microencapsulated cells survive at least two weeks after preparation in vitro. Monocytes produce growth factors, such as vascular endothelial growth factor (VEGF). VEGF is a homodimeric glycoprotein of relative molecular mass of 45,000 Da [17]. Therefore, to achieve study the release of the growth factor, such us VEFG, secreted from the cells immobilized inside the microcapsules, is sufficient to study the release of RBID.

\subsection{Effect of Alginate Concentration in the Cumulative Release of RBID}

Also, Figures 5 and 6 shows that high alginate concentration $(3.0 \mathrm{wt} \%)$ leads to a higher cumulative release. This behaviour is due to the mechanism of formation of microcapsules. Once the alginate-RBID drop takes contact with the cationic solution, instantaneously starting membrane formation.

An increase of the alginate concentration produces an increase of the number of molecules of the biopolymer per volume unit available to react with the divalent cations of barium chloride, producing quickly a very dense network of gel [18]. As a result, there will be fewer available alginate effective charges to interact with the RBID molecules, leaving more free RBID molecules that are able to release to the supernatant, giving higher values of cumulative release.

\subsection{Effect of Forced Convection in the Cumulative Release of RBID}

In this section it is compared the experimental data from the steady and unsteady processes to observe the effect of the forced convection in the mass transfer process (Table 4). As shown in the table, the values of cumulative release of RBID in the unsteady process, for all the variables studied, are higher that those of the steady process.

In the unsteady mass transfer process, besides the mass transfer by diffusion, the fluid supplied by the peristaltic pumps produces advection processes removing more quantity of RBID from capsules because it has a double effect. The fluid is continuously making the RBID coming out of the capsules, but also renews the
Tabla 4. Cumulative release data.

\begin{tabular}{ccccc}
\hline & \multicolumn{2}{c}{$\begin{array}{c}\text { Steady mass } \\
\text { transfer study }\end{array}$} & \multicolumn{2}{c}{$\begin{array}{c}\text { Unsteady mass } \\
\text { transfer study }\end{array}$} \\
\cline { 2 - 5 } $\begin{array}{c}\text { Molecular weight of } \\
\text { RBID (KDa) }\end{array}$ & \multicolumn{4}{c}{ Alginate concentration (wt\%) } \\
\cline { 2 - 5 } & $\mathbf{1 . 2}$ & $\mathbf{3 . 0}$ & $\mathbf{1 . 2}$ & $\mathbf{3 . 0}$ \\
\cline { 2 - 5 } & \multicolumn{4}{c}{ Cumulative release, $\mathbf{M} / \mathbf{M}_{\infty}$ (\%) } \\
\hline $\mathbf{1 0}$ & 28 & 31 & 44 & 54 \\
$\mathbf{4 0}$ & 49 & 61 & 67 & 90 \\
$\mathbf{7 0}$ & 52 & 57 & 68 & 92 \\
\hline
\end{tabular}

supernatant constantly, so that, the concentration of RBID in the supernatant is maintained at zero, producing a constant increase of the concentration gradient between the capsule and supernatant.

\section{Theoretical Mass Transfer Study}

Due to the difficulties encountered in the validation of the mass transfer in patients (in vivo), modelling plays an important role to find a suitable release profile to optimize the therapeutic efficacy of a substance immobilized [11].

For these reasons, in this paper, based on the experimental work, a theoretical model was developed to simulate the mass transfer processes of the release of a protein from capsules to different conditions.

\subsection{Theoretical Steady Mass Transfer Study}

Based on the work of Lewinska et al. [19], a mathematical model was developed in order to simulate the steady mass transfer of the drug from capsules into a finite external volume, that is, a steady mass transfer process.

Several assumptions were taking into account:

1) Buoyancy/gravity effects in the fluid are neglected.

2) The initial concentration of the tracer in the surrounding solution is zero.

3) There is not a concentration gradient inside the sphere.

The last assumption implies that it is considered each sphere as an ideal well-mixed batch reactor with an identical tracer concentration $C_{S}$ everywhere inside a capsule at a given moment of time.

A schematic of the process is described in Figure 10, where before the beginning of the mass transfer process, all the RBID molecules are inside of the capsules, that is, the concentration of RBID in the capsules, $C_{S}$, is the same that the initial concentration, $C_{0}$. Without any loss of generality, it is considered that the initial concentration of RBID in the supernatant is zero (initially "RBID-free" medium, $C=0$ ). At the inception of the process $(t=0)$, RBID starts diffusing out from the capsule, that is, the steady mass transfer process starts from 


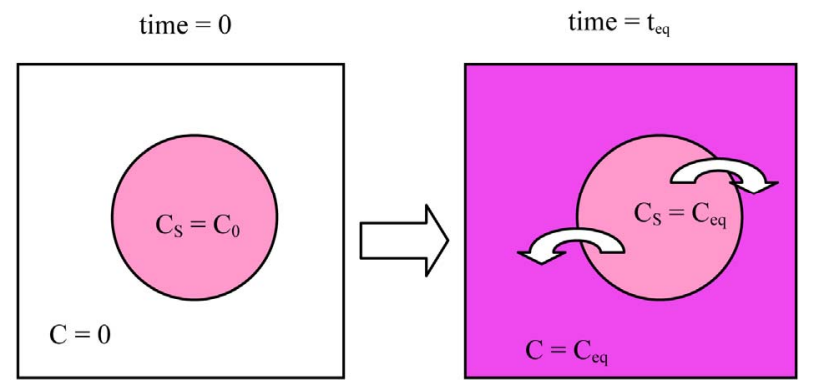

Figure 10. Initial conditions and time system evolution.

the capsule to the supernatant of the reactor, where RBID will be accumulating with time until the equilibrium state is achieved $\left(t=t_{e q}\right)$ with a equilibrium concentration, $C_{e q}$. In this moment the concentration of RBID in the capsule and in the supernatant will be the same and the mass transfer process will stop.

The mass transfer process from the capsules to the supernatant can be generally described [20] by Equation (2), where the variation of the concentration in the supernatant is due to the mass transfer from the capsules to the supernatant:

$$
V_{0} \frac{\mathrm{d} C}{\mathrm{~d} t}=-h A\left(C_{S}-C\right)
$$

where $C$ is the concentration of the tracer in solution outside capsules, and $C_{S}$ the concentration of the tracer inside capsules. The symbol $A$ designates the total external surface area of capsules, with $h$ representing the mass transfer coefficient, $V_{0}$ the volume of solution outside capsules, and $V_{C}$ the volume of capsules. The mass transfer coefficient, $h$, describes the resistance to mass transfer, $1 / h$, in the fluid boundary layer at the surface of the sphere. The driving force for mass transfer is the concentration gradient of the tracer in the batch reactor.

Taking into account the following boundary condition: in the equilibrium state there is a constant equilibrium concentration, $C_{e q}$, inside and outside the capsules, $C_{S}(t=\infty)=C(t=\infty)=C_{e q}$, the Equation (2) can be complemented by a mass balance Equation (3) in the form:

$$
V_{0} C+V_{C} C_{S}=\left(V_{0}+V_{C}\right) C_{e q}
$$

Then, the direct relationship between concentrations $C$ and $C_{S}$ is represented by Equation (4):

$$
C=\left(\frac{V_{0}+V_{C}}{V_{0}}\right) C_{e q}-\frac{V_{C}}{V_{0}} C_{S}
$$

The substitution of $C$ (Equation 4) into Equation (2) and solution of this resultant equation with the initial condition $C_{S}(t=0)=C_{0}$, yields a general expression describing the RBID concentration $C_{S}$ inside the capsules as a function of time, given by Equation (5):

$$
\frac{C_{S}}{C_{0}}=\frac{C_{e q}}{C_{0}}+\left(1-\frac{C_{e q}}{C_{0}}\right) \mathrm{e}^{\left[-\left(\frac{1}{V_{0}}+\frac{V_{C}}{V_{0}^{2}}\right) h A t\right]}
$$

The value of $V_{0}$ is known for a given experiment as well as the total capsule volume $V_{C}$. Knowing the experimental average capsule diameter $(D)$ in a sample, by an optical microscope (Leica DM 1000), the total external surface area of capsules $(A)$ can be approximated from their geometrical surface. For the capsules with small size dispersion (less than 10\%), samples can be represented by $N$ spheres of diameter $\left(d_{p}\right)$ and their total surface area $(A)$ can be expressed as:

$$
A=N\left(4 \pi\left(\frac{d_{p}}{2}\right)^{2}\right)
$$

The total capsule volume $V_{C}$ can be estimated as:

$$
V_{C}=N\left(\frac{4}{3} \pi\left(\frac{d_{p}}{2}\right)^{3}\right)
$$

The experimental data corresponding to the cumulative release profiles were correlated using Mathematica ${ }^{T M}$ computer program, according to Equation (5), using the mass transfer coefficient, $h$, in the model as a fitting parameter. The mass transfer coefficient values, $h$, were obtained for the different conditions, and were collected in Table 5. Also, the values of the effective diffusivity, calculated from the values of the mass transfer coefficient, h, and the Sherwood number (Explanation Table 1) $[21,22]$ were listed in Table 5 to observe the effect of the diffusion in the overall process.

From Table 5 it can be observed that the values of both the external mass transfer coefficient and the effective diffusivity decrease when the molecular weight of RBID and the alginate concentration increase, that is, the mass transfer rate decrease.

The effective diffusion coefficients given in Table 5 refer to the effective diffusion of the RBID in the capsules structure that is different to that obtained in solu-

\begin{tabular}{|c|c|c|c|c|}
\hline \multirow{3}{*}{$\begin{array}{c}\text { Molecular } \\
\text { weight of } \\
\text { RBID (Da) }\end{array}$} & \multicolumn{4}{|c|}{ Alginate concentration (wt $\%)$} \\
\hline & 1.2 & 3.0 & 1.2 & 3.0 \\
\hline & \multicolumn{2}{|c|}{$\begin{array}{c}\text { Mass transfer coefficient, } \\
h(\mathrm{~cm} / \mathrm{s})\end{array}$} & \multicolumn{2}{|c|}{$\begin{array}{l}\text { Effective diffusivity, } D_{e}, \\
\left(\mathrm{~cm}^{2} / \mathrm{s}\right)\end{array}$} \\
\hline 10000 & $3.10 \times 10^{-4}$ & $9.62 \times 10^{-5}$ & $2.12 \times 10^{-8}$ & $4.69 \times 10^{-9}$ \\
\hline 40000 & $1.66 \times 10^{-4}$ & $3.76 \times 10^{-5}$ & $8.33 \times 10^{-9}$ & $1.15 \times 10^{-9}$ \\
\hline 70000 & $1.43 \times 10^{-4}$ & $3.20 \times 10^{-5}$ & $6.66 \times 10^{-9}$ & $9.00 \times 10^{-1}$ \\
\hline
\end{tabular}
tion. In the case of this paper, RBID is not free, but inside the crosslinked gel network.

In comparison, data of diffusion coefficients obtained

Table 5. Values of mass transfer coefficients and effective diffusivity at different conditions (steady process). 
by Koutsopoulos et al. [23] to the controlled release of different proteins from croslinked gel networks and solution are attached in Table 6, including an estimation of the Brownian diffusion by the Stokes-Einstein equation.

Effect of molecular weight of RBID in the mass transfer coefficient, $h$, and the effective diffusivity, $D_{e}$ :

The values of both the external mass transfer coefficient and the effective diffusivity decrease when the molecular weight of RBID increases, that is, the mass transfer rate decreases with a higher RIBD molecular weight, because there is more resistance to the mass transfer.

In the case of high molecular weight of RBID, the free molecules of RBID have a great difficulty to get out through the pores of the capsules due to the steric hindrance of the large dextran molecules, hence the lower transfer rate. When the molecular weight of RBID decrease, the steric hindrance decrease, and then the rate of release of the free RBID molecules will be higher, since there is less resistance to mass transfer.

The effective diffusion coefficient, De, must decrease with increasing the size of molecules, since the equation that describes Brownian diffusion-the Stokes Einstein equation (Equation (8)) - shows that the size of the molecules is indirectly proportional to the Brownian diffusion coefficient [24], as can be observed in the values obtained in this paper, shown in Table 5.

This effect was also reflected in the work of Koutwopulos et al. [23], as shown in Table 6.

$$
D_{b}=\frac{k T}{3 \pi \mu d}
$$

where $k$ is the Boltzmann constant, $T$ is the temperature in Kelvin scale, $\mu$ is the viscosity of the liquid, and $d$ is the diameter of the molecules.
Effect of alginate concentration in the mass transfer coefficient, $h$, and the effective diffusivity, $D_{e}$ :

The values of both the external mass transfer coefficient and the effective diffusivity decreases when the alginate concentration increase, that is, the mass transfer rate decrease with a higher alginate concentration, because there is more resistance to the mass transfer. This behavior is due to the mechanism of formation of microcapsules. Once the alginate-RBID drop takes contact with the cationic solution, the formation of the membrane starts instantaneously. The membrane grows from outside to inside. An increase of the alginate concentration produces an increase of the number of molecules of the biopolymer per volume unit available to react with the divalent cations of barium chloride, producing quickly a very dense network of gel [18]. This dense membrane offers higher resistance to the mass transfer of the RBID molecules, that is, the capsules will have less porosity, decreasing the rate of mass transfer.

In fact, these explanations were proved in a qualitative way, since the capsules formed with a higher concentra-

Table 6. Diffusion coefficients estimated for the release of different proteins at different conditions.

\begin{tabular}{ccccc}
\hline \multirow{2}{*}{ Protein } & Molecular & \multicolumn{3}{c}{ Diffusion coefficients $\left(10^{-10} \mathrm{~m}^{2} / \mathrm{s}\right)$} \\
\cline { 3 - 5 } weight (KDa) & $\begin{array}{c}\text { Estimation (Ec. } \\
\text { Stokes-Einstein) }\end{array}$ & $\begin{array}{c}\text { Effective } \\
\text { diffusion in } \\
\text { solution }\end{array}$ & $\begin{array}{c}\text { Effective } \\
\text { diffusion } \\
\text { through gel }\end{array}$ \\
\hline $\begin{array}{c}\text { Lysozyme } \\
\text { Trypsin } \\
\text { inhibitor }\end{array}$ & 14.3 & 1.15 & 0.96 & 0.50 \\
BSA & 20.1 & 0.91 & 0.72 & 0.32 \\
IgG & 66.0 & 0.61 & 0.51 & 0.24 \\
\hline
\end{tabular}

(Koutsopoulos et al., 2009).

\section{Explanation Table 1. Determination of the effective diffusion coefficient.}

The Sherwood number, $S h$, is a dimensionless number that relates the resistance to mass transfer to the resistance to molecular diffusion, that is, it measures the ratio of the mass transfer flux to the diffusive driving force.

$$
S h=\frac{h d_{p}}{D_{M}}
$$

where $d_{p}$ is the diameter of the capsules, $D_{M}$ is the molecular diffusion coefficient, and $h$ is the mass transfer coefficient.

The Sherwood number can be expressed by means of two dimensionless numbers, the particle Reynolds number, $R e_{p}$, and the Schmidt number, Sc, by using the empirical correlation [27]:

$$
S h=2+1.1 \operatorname{Re}_{p}^{0.6} S c^{\frac{1}{3}} \quad \text { (2) } \quad \operatorname{Re}_{p}=\frac{d_{p} \rho v}{\mu} \quad \text { (3) } \quad S c=\frac{\mu}{\rho D_{M}}
$$

The empirical correlation (2) can be applied to spherical particles with a particle Reynolds number between 3 and 10000 [27]. The molecular diffusion coefficient, $D_{M}$, is determined from Equations (1-4).

The effective diffusion coefficient, $D_{e}$, is obtained from the molecular diffusion coefficient, $D_{M}$, and the porosity, $\varepsilon$, taking into the account that each capsule is immersed in a packed bed formed by the other capsules, resulting in an isotropic porous medium. According to the model of Wakao and Smith [28], the effective diffusion coefficient may be calculated from equation (5):

$$
D_{e}=D_{M} \varepsilon^{2} \quad \text { (5) } \quad \varepsilon=\frac{V_{\text {empy }}}{V_{\text {total }}}=\frac{V_{\text {total }}-V_{\text {spheres }}}{V_{\text {total }}}=0.82
$$


tion of alginate were more resistant from a mechanical point of view, due to the dense structure of the gel.

Table 6 [23] shows the values of the diffusion coefficients to different proteins determined both in solution and through a gel, and through the Stokes-Einstein equation. The results show that the Stokes-Einstein equation slightly overestimates the diffusivities of the proteins in solution by $10 \%-20 \%$, but differed significantly from those calculated through the gel. This means that the Brownian diffusion predicts the behaviour of networks of gels of very low density that can be likened to the release of a protein in solution. It is noticed that the diffusion coefficients calculated for the diffusion of proteins through a polymer network are much lower than those calculated for the diffusion of the protein in solution. This reflects the importance of the grade of crosslink of the gel network in the diffusion coefficient, that is, the denser the network of the gel, the lower the diffusion coefficient, as there will be greater resistance to diffusion. This behaviour reflected in the data of Koutsopoulos et al. [23] can be also observed in the results presented in this paper in the Table 5, that is, the effective diffusion coefficient decreases with increasing concentration of alginate that increases the density of the gel network.

\subsection{Theoretical Unsteady Mass Transfer Study}

Macroscopically, the tissue is ideally assumed as an isotropic porous medium, which is described by Darcy's law [11].

Based on the Feng and Michaelides [25] mass transfer studies, in order to develop a mathematical model to simulate the unsteady mass transfer of a protein from a microcapsule in a porous medium, the following assumptions were considered:

1) Buoyancy/gravity effects in the fluid are neglected.

2) It is considered a constant diameter of the microcapsules with time (no shrink).

3) There is not a concentration gradient inside the sphere.

4) Velocity field inside the porous medium is governed by Darcy's law and is unidirectional far from the sphere.

5) Torsional flow.

6) The initial tracer concentration on the bulk fluid is zero.

7) The velocity field around the spheres is a solution to the potential flow.

The assumption 3) implies that each sphere is considered as an ideal well-mixed batch reactor where the concentration at any point in the sphere is the same.

The 4) assumption implies that there may be slip at the surface of the sphere. However, here the Brinkman model - extended Darcy formulation with inertial term included - that takes into account the no slip condition on the surface of the sphere, has been applied, because in earlier works it has been established that the pure Darcy law does not give satisfactory results when it is required to take into account the no slip boundary condition [26]. However, after several considerations it is possible to conclude that the thickness of the boundary layer is a millionth of the sphere's radius. Inside the boundary layer, there is a very high velocity gradient in the tangential direction, with the velocity increasing rapidly from zero to the value calculated by Darcy's law. Because of the very small relative thickness of the boundary layer, it is acceptable to neglect it and to consider that there is a step velocity change from zero (no slip condition) to Darcy's slip velocity in the tangential direction to the surface [25].

A schematic of the process and of the coordinate system is depicted in Figure 11.

The presence of the sphere creates a disturbance to this velocity field, which is essentially confined to the vicinity of the sphere. Far from the sphere, the velocity field is unidirectional. At the inception of the process $(t=0)$, RBID starts leaking from the sphere. The two fluids mix freely and are transported in the porous medium. Without any loss of generality, it is considered that the initial concentration of RBID in the porous medium is zero (initially "RBID-free" medium). After the inception of the leakage process $(t>0)$ it should be considerer a boundary condition at the surface of the sphere: the concentration of RBID is constant, $C_{s 0}$.

Then, the boundary conditions for the external fluid are:

$$
\begin{array}{ll}
v \cdot n=0 & \text { at } \quad r=a \\
v=-U \cdot \mathrm{e}_{z} \quad \text { at } & r \rightarrow \infty
\end{array}
$$

From the velocity field in the porous medium and using the boundary conditions for the external fluid, which initially saturates the porous medium, at the surface/infinite, it is obtained the velocity field of the capsules that is a solution to the potential flow problem.

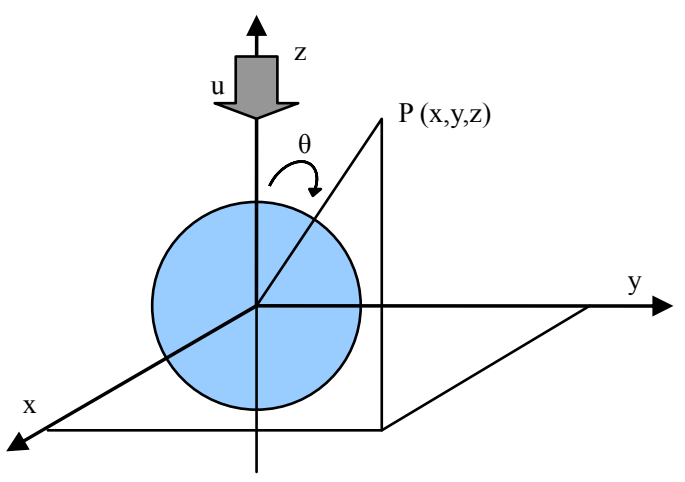

Figure 11. The flow and the coordinate system. 
That is [27],

$$
\begin{gathered}
v_{r}=U_{\infty}\left[1-\left(\frac{a}{r}\right)^{3}\right] \cos \theta \\
v_{\theta}=-U_{\infty}\left[1+\frac{1}{2}\left(\frac{a}{r}\right)^{3}\right] \sin \theta
\end{gathered}
$$

For convenience, it is introduced the following dimensionless variables, which are denoted by an asterisk $(*)$. It is considered as the characteristic length of the process the radius of the sphere, $a$, and as characteristic time the diffusion time scale, $\tau_{D}=a^{2} / D_{e}$ :

$$
\begin{aligned}
& \left(x^{*}, y^{*}, z^{*}\right)=\left(\frac{x}{a}, \frac{y}{a}, \frac{z}{a}\right), \quad r^{*}=\frac{r}{a}, \\
& t^{*}=\frac{t D_{e}}{a^{2}}, c^{*}\left(x^{*}, t^{*}\right)=\frac{c(x, t)}{C_{s 0}}, \\
& v^{*}=\frac{v}{U_{\infty}}, \quad P e=\frac{U_{\infty} a}{D_{e}}
\end{aligned}
$$

It is noteworthy the Peclet number that is a dimensionless number relating the rate of advection of a flow to its rate of diffusion

Upon substitution into (10) and (11), the following dimensionless velocity field is obtained in the spherical coordinates:

$$
\begin{aligned}
& v_{r}^{*}=-\left[1-\frac{1}{\left(r^{*}\right)^{3}}\right] \cos \theta \\
& v_{\theta}^{*}=\left[1+\frac{1}{2\left(r^{*}\right)^{3}}\right] \sin \theta
\end{aligned}
$$

$U_{\infty}$ is the fluid velocity far from the sphere. However, as it is intended to simulate real living systems (e.g heart is in a continuous contraction-relaxing movement) it was introduced stirring to the system by an orbital agitator, for this reason it is assumed torsional flow on the main assumptions. Torsional flows are induced by rotating solid boundaries in contact with liquids. The liquid, due to the no-slip boundary condition, has to follow the motion of the boundary, and therefore a torsional flow is generated. Then, $U_{\infty}$, the fluid velocity far from the sphere will be calculated by adding the flow velocity through the porous medium supplied by the pumps to the velocity due to the torsional flow supplied by the velocity of the orbital agitator.

The macroscopic quantity of the velocity due to the torsional flow can be calculated as the following [28]:

$$
\bar{V}=R^{2} \Omega z_{0}+\frac{4 R^{4} \Omega^{3}}{g}
$$

The governing equation of the tracer transport process has to be expressed in dimensionless form [29]:

$$
\frac{\partial c^{*}}{\partial t^{*}}+P e v^{*} \cdot \nabla^{*} c^{*}=\nabla^{* 2} c^{*}
$$

with the dimensionless initial and boundary conditions defined as follows:

$$
\begin{aligned}
& c^{*}\left(x^{*}, t^{*}\right)=0 \quad \text { at } \quad|x *|>1, \quad t^{*}=0 \\
& c^{*}\left(x^{*}, t^{*}\right)=H\left(t^{*}\right) \text { at }\left|x^{*}\right|=1, \quad t^{*}>0 \\
& c^{*}\left(x^{*}, t^{*}\right) \rightarrow 0 \quad \text { as } \quad\left|x^{*}\right| \rightarrow \infty .
\end{aligned}
$$

Equations (13)-(17) showed an unsteady convectiondiffusion problem.

For convenience, in the equations that follow, it will be omitted the superscript $*$ of the dimensionless variables. It must be remembered, however, that the variables used hitherto are dimensionless.

As the experimental results show the mass transport process is taken about 100 minutes and considering the average time of this kind of processes is in the range of hours, therefore this process could be considerer as a short-time process. The solution in this type of process is constructed by satisfying the boundary conditions at the surface of the sphere and at infinity.

The concentration of the tracer in the fluid may be given by a regular expansion of the concentration function $c(x, t)$ as follows [25]:

$$
c=c_{0}+P e \cdot c_{1}+\cdots
$$

A first order expansion of the concentration function is sufficient for the development of the solution.

The final analytical expression for RBID concentration is obtained by solving the governing equation in the time domain or the Laplace domain [25]:

$$
\begin{aligned}
c(r, \zeta, t)= & \frac{1}{r} \operatorname{erfc}\left(\frac{r-1}{2 \sqrt{t}}\right) \\
& +P e\left[\left(-\frac{1}{2}+\frac{3}{4 r^{2}}-\frac{1}{4 r^{3}}\right) \operatorname{erfc}\left(\frac{r-1}{2 \sqrt{t}}\right)\right. \\
& \left.+\frac{3}{4}\left(\frac{1}{r}-\frac{1}{r^{2}}\right) \mathrm{e}^{r-1+t} \operatorname{erfc}\left(\frac{r-1}{2 \sqrt{t}}+\sqrt{t}\right)\right] P_{1}(\zeta)
\end{aligned}
$$

For the practical applications of this mass transfer problem, i.e. the repair of tissues (cell therapy), is important to know information about the distribution of the concentration of the substance immobilized around the capsules throughout the diffusion process to a certain distance of the application point to be able to design therapies to treat different diseases.

From the model described by Equation (19) it is possible to generate a prediction of the concentration distri- 
bution fields as a function of the radius, the angular coordinate, at different times. To make that, the model equation was represented in parametric coordinates, and the dimensionless radius was varied continuously from 0 to 3 in both sides of the capsule. The initial concentration condition is a unit step change at the surface of the sphere [25]. This corresponds to the process of the leakage at $t=0$. In order to generate the concentration distributions were set different Peclet numbers $\left(\mathrm{Pe}=\mathrm{U}_{\infty} \mathrm{a} / \mathrm{D}_{\mathrm{e}}\right)$ that takes into account the relationship between the process of convection and diffusion. Based on the velocity data of the peristaltic pumps, and the effective diffusivity data previously obtained in the steady process, it was set the different Peclet numbers to each experimental condition.

Figure 12 shows the concentration distribution field, which results for a molecular weight of RBID of 10000 and an alginate concentration of $1.2 \mathrm{wt} \%$.

From Figure 12 it is possible to observe that very close to the surface also of the sphere $(r<1.2)$ the concentration profile does not vary significantly during the process of mass transfer.

However, there is an appreciable change in concentration profile far from the surface $(r<1.5)$. This is an indication that an almost equilibrium state is quickly established in the immediate vicinity of the sphere. However, the RBID migrates at a faster rate towards the outer region due to the influence of the peristaltic pumps. The aspect of the Figure 12 (from top to bottom) underlines the small significance of the advection with respect to the diffusion process.

Figure 13 shows the concentration distribution field, which results for a molecular weight of RBID of 70,000 and an alginate concentration of $1.2 \mathrm{wt} \%$.

From Figure $\mathbf{1 3}$ it is possible to observe that the concentration gradient do not vary significantly from left to right (y direction) during the process of mass transfer. However, it is shown a large concentration gradient from top to bottom (flow direction, $\mathrm{z}$ ) since the inception of the mass transfer process. RBID spread out in the flow direction while not in the opposite direction. This shows the importance of the advection with respect to the diffusion process at high molecular weight of RIBD. A cumulative release increase has to be produced by a reduction of the rate of diffusion to increase the advection effect with regard to the diffusion effect.

From Figure 14 it is possible to observe the evolution of the distribution profiles with increasing molecular weight of RBID. It can be observed an increase in the contribution of convection in the overall mass transfer process when it is increased the molecular weight of RBID.

It was shown that there is an effect of the molecular weigh of the protein on the cumulate release. The smallest molecules exhibit a much lower cumulative release. This behavior can be assigned to the presence of attrac-

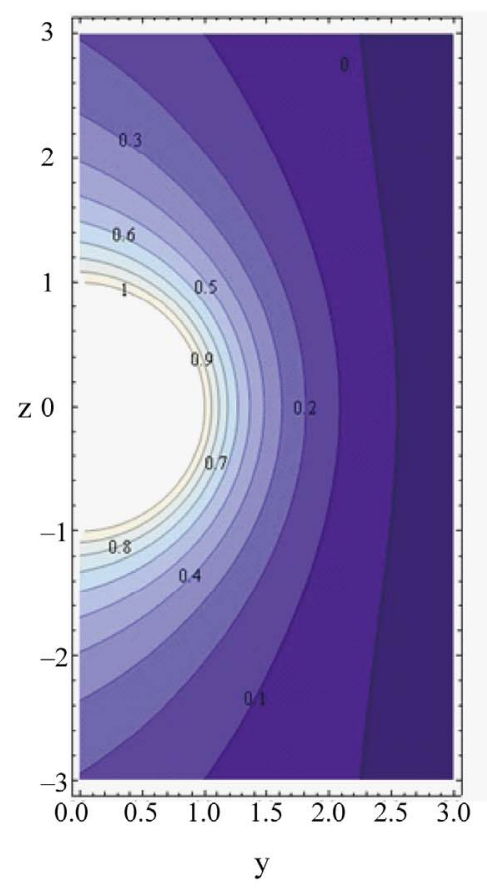

(a)

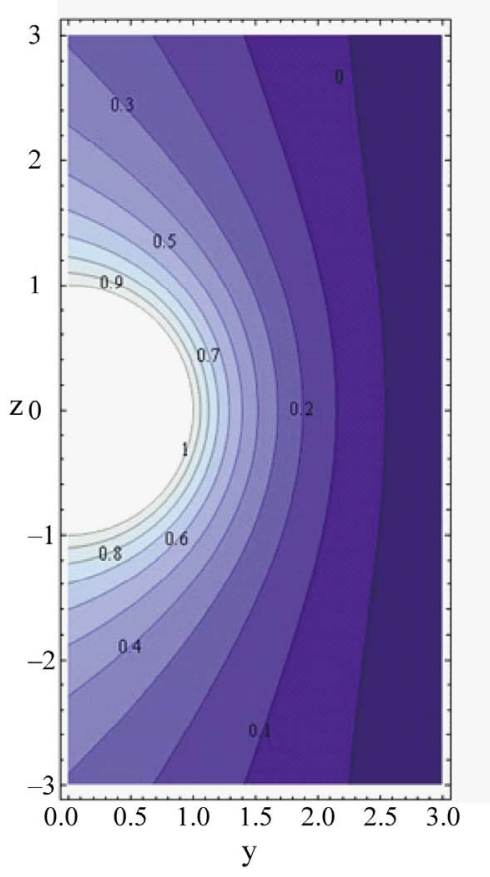

(b)

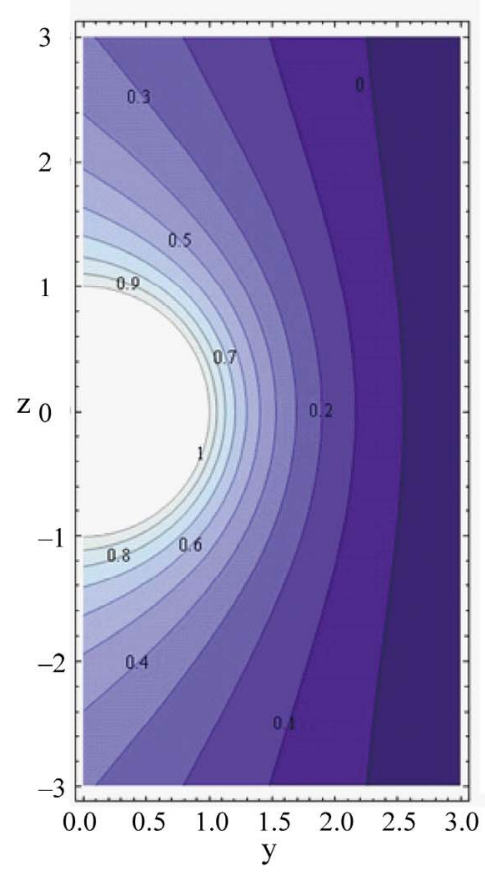

(c)

Figure 12. Concentration distribution profiles (RBID MW = 10000; alginate concent. 1.2 wt\%). (a) 2 minutes; (b) 22 minutes; (c) 99 minutes. 


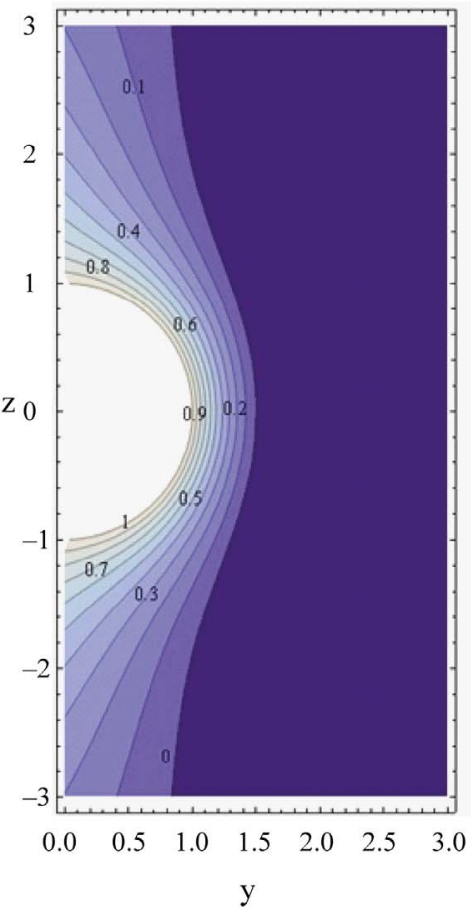

(a)

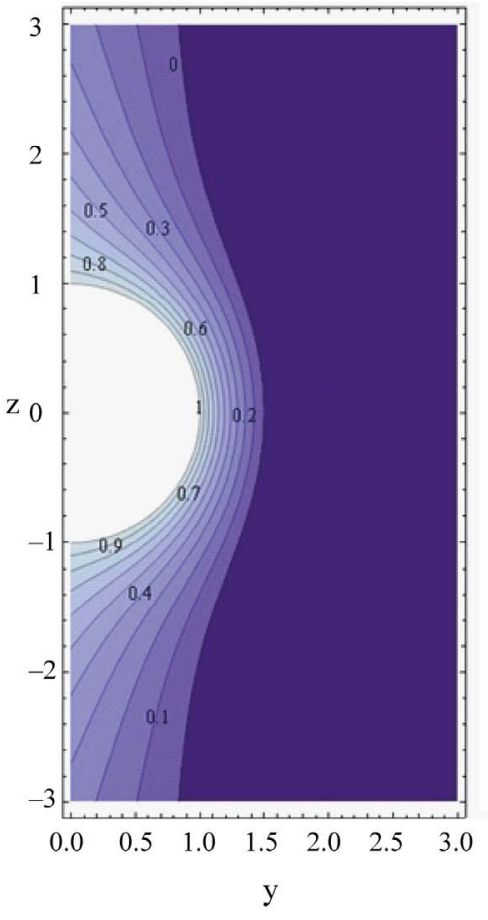

(b)

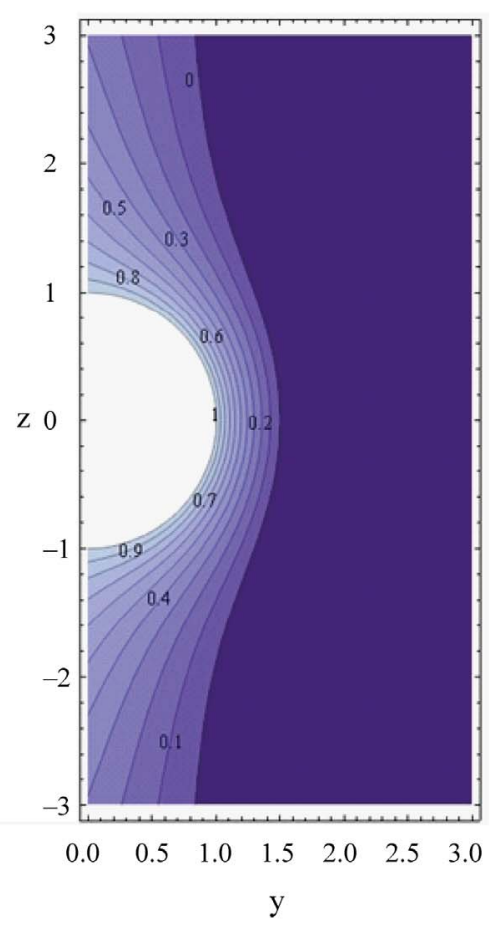

(c)

Figure 13. Concentration distribution profiles (RBID MW = 70000; alginate concent. 1.2 wt\%). (a) 6 minutes; (b) 22 minutes; (c) 99 minutes.

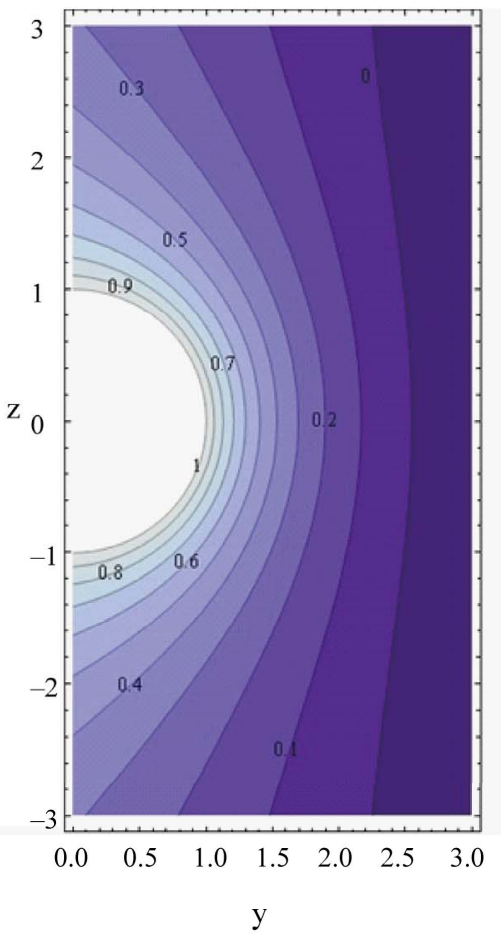

(a)

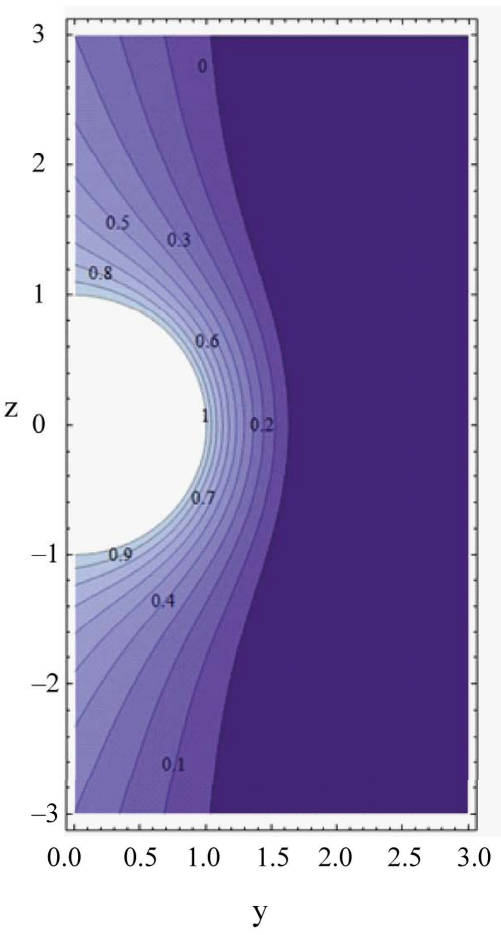

(b)

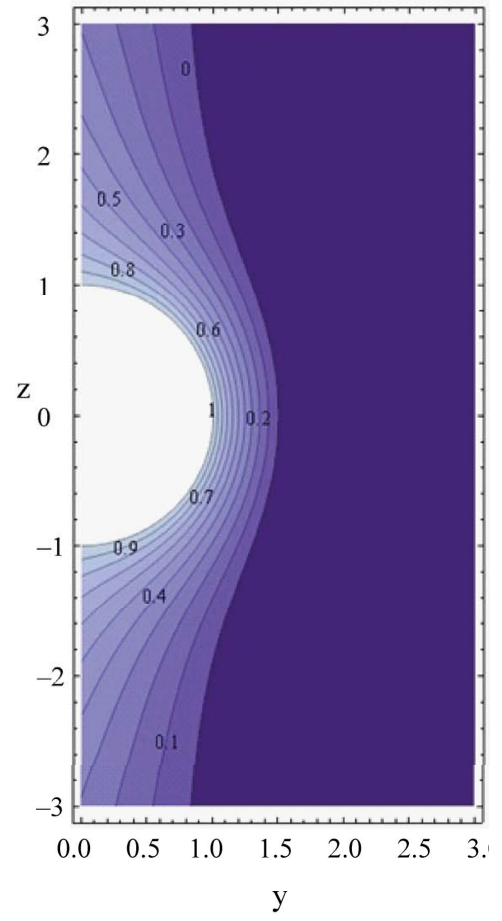

(c)

Figure 14. Concentration distribution profiles (Time $=88$ minutes/alginate concentration $=1.2$ wt\%). RBID MW (a) 10000 Da; (b) 40000 Da; (c) 70000 Da. 
tive electrostatic interactions between protein and alginate. Due to the amphoteric character, at higher $\mathrm{pH}$ values, the attraction of protein to the alginate may be diminished as a result of unfavorable electrostatics interactions. Knowing this, it is possible to achieve a pH-dependent controlled release of proteins, resulting in protein imprinting processes. In particular, it was mimicked the behaviour of a special type of proteins, glycoproteins.

Based on the experimental work, and several theoretical mass transfer studies, it was predicted the coefficients of the mass transfer process to the steady and unsteady processes.

From the steady mass transfer study it can be concluded that the rate of mass transfer decreases when the molecular weight of RBID increases due to the size of the RBID molecules that have more difficulties to be released through the pores of the capsules. Also, the rate of mass transfer decreases when the alginate concentration increases due to the mechanism of formation of the capsules.

\section{Conclusions}

From the experiments reported here it can be concluded that electrostatic interactions play a very important role on the release of proteins from alginate-barium capsules.

From the final analytical expression for the unsteady mass transfer it is possible to predict the concentration fields around the capsules as a function of the angular coordinate, the radius and the time. From the concentration distribution field to low molecular weight of RBID it is possible to observe that an almost equilibriumstate condition is quickly established in the immediate vicinity of the sphere, but the active migrates with a faster rate towards the outer region. However, the concentration distribution field to high molecular weight of RBID shows the importance of the advection with respect to the diffusion process. A cumulative release increase has to be produced by a reduction of the rate of diffusion to increase the advection effect with regard to the diffusion effect.

\section{Acknowledgements}

The authors gratefully acknowledge Prof. Dr. D. Jose Ángel Dominguez from the Mathematics Department of the University of Salamanca for the help with Mathematica $^{\mathrm{TM}}$. This research was supported by funds from the Ministry of Science and Education (MEC), Junta de Castilla y León, and the European Research Council (ERC-Starting grant 2010, IP: Eva M. Martín del Valle, Project MYCAP). The authors gratefully acknowledge the financial support.

\section{References}

[1] C. Dai, B. Wang and H. Zhao, "Microencapsulation Peptide and Protein Drugs Delivery System," Colloids and Surfaces B: Biointerfaces, Vol. 41, No. 2-3, 2005, pp. 117-120. doi:10.1016/j.colsurfb.2004.10.032

[2] E. M. M. Del Valle, M. A. Galan and R. G. Carbonell, "Drug Delivery Technologies: The Way Forward in the New Decade", Industrial \& Engineering Chemistry Research, Vol. 48, No. 5, 2009, pp. 2475-2486. doi: $10.1021 /$ ie $800886 \mathrm{~m}$

[3] G. A. Peyman and G. J. Ganiban, "Delivery Systems for Intraocular Routes," Advanced Drug Delivery Reviews, Vol. 16, No. 1, 1995, pp. 107-123. doi:10.1016/0169-409X(95)00018-3

[4] H. Kimura, et al., "Injectable Microspheres with Controlled Drug Release for Glaucoma Filtering Surgery," Investigative Ophthalmology and Visual Science, Vol. 33, No. 12, 1992, pp. 3436-3441.

[5] D. F. Martin, "Treatment of Cytomegalovirus Retinitis with an Intraocular Sustained Release Ganciclovir Implant: A Randomized Controlled Clinical Trail," Archives of Ophthalmology, Vol. 112, No. 12, 1994, pp. 15311539.

[6] M. Otsuka, et al., "A Novel Skeletal Drug Delivery System Using a Self-Setting Calcium Phosphate Cement. 4. Effects of the Mixing Solution Volume on the Drug Release Rate of Heterogeneous Aspirin-Loaded Cement," Journal of Pharmaceutical Sciences, Vol. 83, No. 2, 1994, pp. 259-268. doi:10.1002/jps.2600830230

[7] M. Otsuka, “A Novel Skeletal Drug Delivery System Using a Self-Setting Calcium Phosphate Cement. 5. Drug Release Behaviour from a Heterogeneous Drug Loaded Cement Containing an Anticancer Drug," Journal of Pharmaceutical Sciences, Vol. 83, No. 11, 1994, pp. 15651568. doi:10.1002/jps.2600831109

[8] I. G. Needleman, "Controlled Drug Release in Periodontics: A Review of New Therapies," British Dental Journal, Vol. 170, 1991, pp. 405-407. doi:10.1038/sj.bdj.4807569

[9] T. E. Rams and J. Slots, "Local Delivery of Antimicrobial Agents in the Periodontal Pocket," Periodontalogy 2000, Vol. 10, No. 1, 1996, pp. 139-159.

[10] G. Greenstein and A. Polson, "The Role of Local Drug Delivery in the Management of Periodontal Diseases: A Comprehensive Review," The Journal of periodontology, Vol. 69, No. 5, 1998, pp. 507-520.

[11] D. Y. Arifin, et al., "Mathematical Modelling and Simulation of Drug Release from Microspheres: Implications to Drug Delivery Systems," Advanced Drug Delivery Reviews, Vol. 58, No. 12-13, 2006, pp. 1274-1325. doi:10.1016/j.addr.2006.09.007

[12] L. Cumbal, A. K. SanGupta, J. Greenlead and D. Leun, "Polymer Supported Subcolloidal Particles: Characterization and Environmental Application,” In: S. Barany, Ed., 
Role of Interfaces in Environmental Protection, Kluwer Academics Publishers, Dordrecht, 2003.

[13] R. P. Haugland, "The Handbook-A Guide to Florescent Probes and Labelling Technologies," Molecular Proves Inc., Invitrogen, 2005.

[14] P. Peng, N. H. Voelcker, S. Kumar and H. J. Griesser, "Nanoscale Eluting Coatings Based on Alginate/Chitosan Hydrogels," Bioinerphases, Vol. 2, No. 2, 2007, pp. 95104. doi:10.1116/1.2751126

[15] T. Kawaguchi and M. Hasegawa, "Structure of Dextran-Magnetite Complex: Relation between Conformation of Dextran Chains Covering Core and Its Molecular Weight," Journal of Materials Science, Vol. 11, No. 1, 2000, pp. 31-35. doi:10.1023/A:1008933601813

[16] E. P. Herrero, E. M. M. Del Valle and M. A. Galan, "Immobilization of Mesenchymal Stem Cells and Monocytes in Biocompatible Microcapsules to Cell Therapy," Biotechnology Progress, Vol. 23, No. 4, 2007, pp. 940945.

[17] J. L. Sharon and D. A. Puleo, "Immobilization of Glycoproteins, such as VEGF, on Biodegradable Substrates," Acta Biomaterialia, Vol. 4, No. 4, 2008, pp. 1016-1023. doi:10.1016/j.actbio.2008.02.017

[18] A. Blandino, M. Macias and D. Cantero, "Glucose Oxidase Release from Calcium Alginate Gel Capsules," Enzyme Microbial Technology, Vol. 27, No. 3-5, 2000, pp. 319-324. doi:10.1016/S0141-0229(00)00204-0

[19] D. Lewinska, et al., "Mass Transfer Coefficient in Characterization of Gel Beads and Microcapsules," Journal of Membrane Science, Vol. 209, No. 2, 2002, pp. 533-540. doi:10.1016/S0376-7388(02)00370-8

[20] D. F. Radeliffe and J. D. S. Gaylor, "Sorption Kinetics in Haemoperfusion Columns. Part. 1. Estimation of Masstransfer Parameters," Medical and Biological Engineering and Computing, Vol. 19, No. 5, 1981, pp. 617-627.
doi:10.1007/BF02442777

[21] N. Wakao and T. Funazkri, "Effect of Fluid Dispersion Coefficients on Particle-to-Fluid Mass Transfer Coefficients in Packed Beds: Correlation of Sherwood Numbers," Chemical Engineering Science, Vol. 33, No. 10, 1978, pp. 1375-1384. doi:10.1016/0009-2509(78)85120-3

[22] N. Wakao and J. M. Smith, "Diffusion in Catalyst Pellets," Chemical Engineering Science, Vol. 17, No. 11, 1962, pp. 825-834. doi:10.1016/0009-2509(62)87015-8

[23] S. Koutsopoulos, L. D. Unsworth, Y. Nagai and S. Zhang, "Controlled Release of Functional Proteins through Designer Self-Assembling Peptide Nanofiber Hydrogel Scaffold," PNAS, Vol. 106, No. 12, 2009, pp. 4623-4628. doi:10.1073/pnas.0807506106

[24] M. Hoyos, "Separación Hidrodinámica de Macromoléculas, Partículas y Células," Acta Biológica Colombiana, Vol. 8, No. 1, 2003, pp. 11-24.

[25] Z.-G. Feng and E. E. Michaelides, "Unsteady Mass Transport from a Sphere Immersed in a Porous Medium at Finite Peclet Numbers," International Journal of Heat Mass and Transfer, Vol. 42, No. 3, 1999, pp. 535-546. doi:10.1016/S0017-9310(98)00160-4

[26] R. Jecl, et al., "Boundary Domain Integral Method for Transport Phenomena in Porous Media," International Journal of Numerical Methods Fluids, Vol. 35, No. 1, 2001, pp. 39-54. doi:10.1002/1097-0363(20010115)35:1<39::AID-FLD81 $>3.0 . \mathrm{CO} ; 2-3$

[27] R. B. Bird, W. E. Stewart and E. N. Lightfoot, "Transport Phenomena,” John Wiley \& Sons, Inc., New York, 2002.

[28] T. C. Papanastasiou, "Applied Fluid Mechanics," PTR Prentice Hall, New Jersey, 1994.

[29] J. Crank, "The Mathematics of Diffusion," Clarendon Press, Oxford, 1975. 


\section{Nomenclature}

\section{Symbol Description}

Radius of the sphere

$a$
$A$

$c$

C

$C_{e q}$

$C_{S}$

$d_{p}$

$D_{e}$

$D_{M}$

$D_{e}$

$e_{r}, e_{z}, e_{\theta}$

erfc

$g$

$h$

K

$n$

$N$

$p$

$P$

$P_{n}$

$\mathrm{Pe}$

$r$

$R e$

$s$

Sc

Sh

$t$

$U$

$v$

$V_{0}$

$x, y, z$

Particle diameter

Effective diffusivity

Unit vectors

Gravity

Permeability

Outward vector

Number of capsules

Pressure

Power input

Peclet number

Radial coordinate

Time variable

Coordinates
Total external surface area of capsules

Concentration function

Concentration of the tracer in solution outside capsules

Concentration in the equilibrium state

Concentration of the tracer inside capsules

Effective diffusion coefficient

Molecular diffusion coefficient

Complementary error function

Mass transfer coefficient

Legendre polynomial

Reynolds Number $\left(d_{p} \rho v / \mu\right)$

variable in Laplace domain to $t$

Schmidt number $\left(\mu / \rho D_{M}\right)$

Sherwood number $\left(h d_{p} / D_{M}\right)$

Fluid velocity far from the sphere

Fluid velocity near the sphere, linear fluid velocity

Volume of solution outside capsules

\section{Units}

$\mathrm{cm}$

$\mathrm{cm}^{2}$

$\mathrm{mg} / \mathrm{ml}$

$\mathrm{mg} / \mathrm{ml}$

$\mathrm{mg} / \mathrm{ml}$

$\mathrm{mg} / \mathrm{ml}$

$\mathrm{cm}$

$\mathrm{cm}^{2} / \mathrm{s}$

$\mathrm{cm}^{2} / \mathrm{s}$

$\mathrm{cm}^{2} / \mathrm{s}$

$\mathrm{m} / \mathrm{s}^{2}$

$\mathrm{cm} / \mathrm{s}$

$\mathrm{m}^{2}$

$\mathrm{N} / \mathrm{m}^{2}$

W

adimensional

$\mathrm{cm}$

adimensional

adimensional

adimensional

$\mathrm{s}$

$\mathrm{cm} / \mathrm{s}$

$\mathrm{cm} / \mathrm{s}$

$\mathrm{cm}^{3}$

$\mathrm{cm}$

$\mu \mathrm{m}$

$\mathrm{kg} / \mathrm{m}^{3}$

radians

adimensional

radians

$\mathrm{kg} / \mathrm{m} \mathrm{s}$ 